\title{
Sediment dynamics on a steep, megatidal, mixed sand-gravel-cobble beach
}

\author{
A. E. Hay ${ }^{1}$, L. Zedel ${ }^{2}$, and N. Stark ${ }^{1, *}$ \\ ${ }^{1}$ Dalhousie University, Department of Oceanography, Halifax, Canada \\ ${ }^{2}$ Memorial University of Newfoundland, Department of Physics and Physical Oceanography, St. John's, Canada \\ *now at: Virginia Tech, Department of Civil and Environmental Engineering, Blacksburg VA, USA
}

Correspondence to: A. Hay (alex.hay@ dal.ca)

Received: 16 January 2014 - Published in Earth Surf. Dynam. Discuss.: 18 February 2014

Revised: 18 July 2014 - Accepted: 24 July 2014 - Published: 21 August 2014

\begin{abstract}
Results are presented from a pilot study of shore-face sediment dynamics on a steep, poorly sorted, coarse-grained, megatidal beach at the head of the Bay of Fundy, Nova Scotia, Canada. The experiment involved the first field deployment of a prototype wideband, pulse-coherent, bistatic acoustic Doppler profiling system. Measurements of the vertical structure of flow and turbulence above a sloping bed, as well as bed material velocity, demonstrate the capabilities of this instrument vis-à-vis studies of nearshore sediment dynamics at the field scale. The second focus of the paper is the unexpected observation that the surficial sediment median diameter, across the lower two-thirds of the intertidal zone, underwent a pronounced decrease when wave forcing was more energetic, compared to values observed during calmer conditions. The explanation for this result appears to involve the formation - in wave-dominated conditions - of metre-scale wavelength, $20 \mathrm{~cm}$ high ripples on the rising tide, which are then planed flat by the swash and/or the shore break on the subsequent ebb.
\end{abstract}

\section{Introduction}

During the past several years we have been developing a prototype wideband acoustic Doppler profiler, the MFDop, for studies of nearshore sediment and fluid dynamics in which the wave bottom boundary layer (WBBL) plays a significant role in the governing dynamics. The instrument, and results from laboratory investigations of turbulent oscillatory flow above fixed-roughness and mobile beds, are described in Hay et al. (2012) and the related articles cited therein. The laboratory experiments served as a proof of concept in preparation for deployment of the instrument in the field. This paper summarizes results from the first field deployment of the MFDop, in April-May 2012.

The experiment was carried out at Advocate Beach, located at the head of the Bay of Fundy. This site was chosen for several reasons. (a) The 10-12 m tidal range allowed the instrument platform to be set up at midtide level on the beach face at low tide, and similarly, the instrument platform could be accessed at low tide to service the instrumentation if required, without the need for diver support. (b) The beach is exposed to the $>500 \mathrm{~km}$ fetch of the Bay of Fundy and Gulf of Maine. (c) The beach is steep (1:10 slope), and measurements of sediment and wave dynamics on steeply sloping beaches are of interest in their own right (Rivero and Arcilla, 1995; Zou et al., 2006).

The beach itself is $5 \mathrm{~km}$ long, and the shoreline is nearly linear. The beach-face sediments range in size from medium sand to $20 \mathrm{~cm}$ diameter and larger cobbles. At low tide, when the beach is fully exposed, the beach face is observed to be uniformly planar, with the exception of remnant cusps at the high and low water marks. The planar section of the exposed profile typically extends from immediately below the berm to the low water line, and can be as much as $100 \mathrm{~m}$ in horizontal extent in spring tides. In surveys conducted over the past 30 years, the beach has remained planar and the 6 to $7^{\circ}$ beach slope has persisted essentially unchanged (Fig. 1). This uniformly planar appearance at low tide is deceptive, however, as we shall demonstrate.

According to the classification scheme introduced by Jennings and Shulmeister (2002), the sand-to-cobble sediment 
size range and the $1: 10$ beach slope correspond to a mixed sand and gravel (MSG) beach. The 10-12 $\mathrm{m}$ tidal range indicates that Advocate Beach can be classed as a megatidal rather than macrotidal beach (Levoy et al., 2000). The reader is referred to the informative reviews by Mason and Coates (2001) of MSG beach dynamics, and by Buscombe and Masselink (2006) of steep and coarse-grained - but pure gravel as opposed to MSG - beaches. Both reviews emphasize the point that there have been far fewer field investigations of flow and sediment dynamics on gravel and MSG beaches than on sand beaches.

Investigating the morphodynamic behaviour of MSG beaches is important for a variety of scientific and coastal management reasons, as outlined in the above reviews. Among the scientific reasons, one worth emphasizing here is the opportunity afforded by the poorly sorted nature of the beach-face sediments to study processes leading to sizesegregation, a topic of increasing interest across a wide range of space- and timescales and sedimentary settings.

During the last decade, results from studies of sediment dynamics on MSG beaches have been reported by Allan et al. (2006), Ivamy and Kench (2006), Ciavola and Castiglione (2009), Curtiss et al. (2009), Dickson et al. (2011), Miller et al. (2011), Bertoni et al. (2012, 2013), and Miller and Warrick (2012). Of these, only Ivamy and Kench (2006) and Curtiss et al. (2009) deployed instruments in the intertidal zone - i.e. where sensors would be subjected to the energetic conditions associated with the shore break. In the remaining studies, sediment movement in the intertidal zone was investigated using tracers alone - either radio-frequency identification tags (Allan et al., 2006; Dickson et al., 2011; Miller et al., 2011; Bertoni et al., 2012, 2013; Miller and Warrick, 2012) or fluorescent paint (Ciavola and Castiglione, 2009) - and information on the wave forcing was obtained from offshore buoys (Allan et al., 2006; Bertoni et al., 2012; Dickson et al., 2011), a pressure sensor in the nearshore either at or beyond the lower low tide level (Miller et al., 2011; Bertoni et al., 2012; Miller and Warrick, 2012; Bertoni et al., 2013), or estimated visually (Ciavola and Castiglione, 2009). Curtiss et al. (2009) deployed acoustic Doppler velocimeters (ADVs) on bottom-mounted tripods in the intertidal, but reported only the peak near-bed velocity registered by these sensors. As far as we have been able to determine, the only study previous to the present work in which sensors have been deployed to measure flow and sediment dynamics in the intertidal zone of an MSG beach is that by Ivamy and Kench (2006, referred to as IK06), who deployed an ADV at the breakpoint. As will be seen, the results from the IK06 experiment are particularly relevant here. However, the sediment dynamics observations in IK06 were limited to active layer thickness measurements with depth-of-disturbance rods, supplemented by pre- and post-experiment bathymetric surveys.

The present paper is concerned with processes operating on storm, tidal and shorter - i.e. intrawave and turbulence -

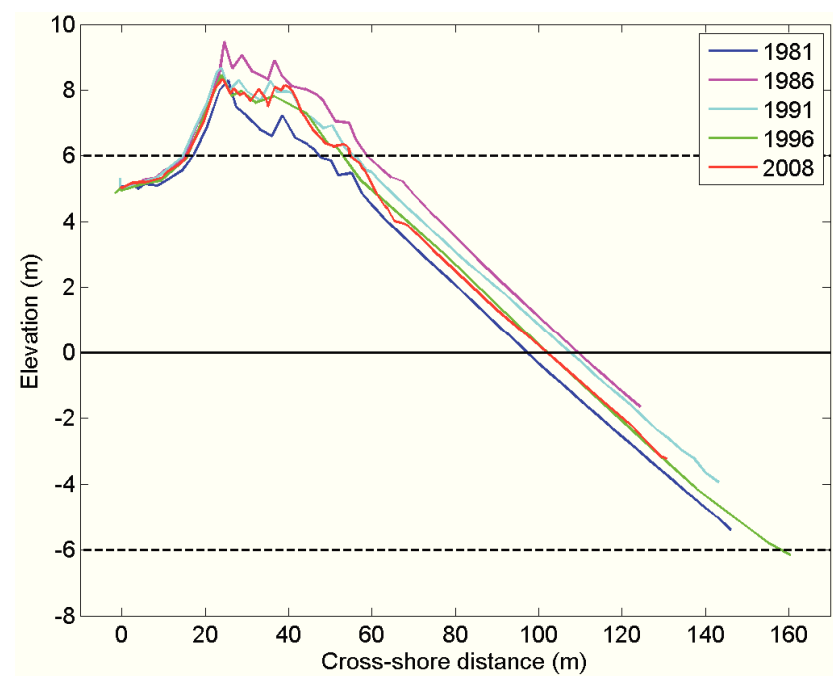

Figure 1. Advocate Beach profile history. Note the 1:10 slope, constant through time. Dashed black lines indicate the nominal low and high tide levels, solid black line the midtide level (data courtesy of Bob Taylor, Natural Resources Canada).

timescales. As such, as far as we have been able to determine from the available literature at least, it is the first comprehensive investigation of flow and sediment dynamics over this range of timescales in the intertidal zone - i.e. the zone affected by the shore break - on an MSG beach.

\section{The Advocate Beach experiment}

The central element of the experiment was the instrument platform shown in Fig. 2. This platform was installed in the beach face at midtide level (Fig. 1, solid black line), and supported the suite of instruments indicated in Fig. 3: (a) rotary sonars to measure the planform geometry and the cross-shore relief of features on the bed; (b) a Nortek Vector ADV to measure wave-current motions outside the WBBL; and (c) the MFDop. The instruments operated on a 15-min repeat cycle. The ADV sample rate was $4 \mathrm{~Hz}$ with a $18 \mathrm{~mm}$ sample volume. The MFDop acquired 10-ping ensemble-averaged profiles with $1.2 \mathrm{~cm}$ range resolution at $52 \mathrm{~Hz}$. The MFDop and ADV record lengths were 4.3 and 4.53 min respectively. The rotary sonars acquired four full rotations with a $0.225^{\circ}$ step size in azimuth and ca. $1 \mathrm{~cm}$ resolution in range. The MFDop and ADV ran simultaneously, and were followed by each rotary sonar in turn, thereby avoiding acoustic interference (the operating acoustic frequencies for the instruments are the MFDop, 1.2-2.3 MHz; the ADV, $6 \mathrm{MHz}$; and the rotary sonars, $2.25 \mathrm{MHz}$ ).

Beach profile surveys and sediment sampling transects were carried out on a regular basis at low tide. Surficial sediment grain-size distributions were determined from discrete $0.4-2.3 \mathrm{~kg}$ sediment samples by dry sieving, and from photographs of the beach face using a tripod-mounted camera 


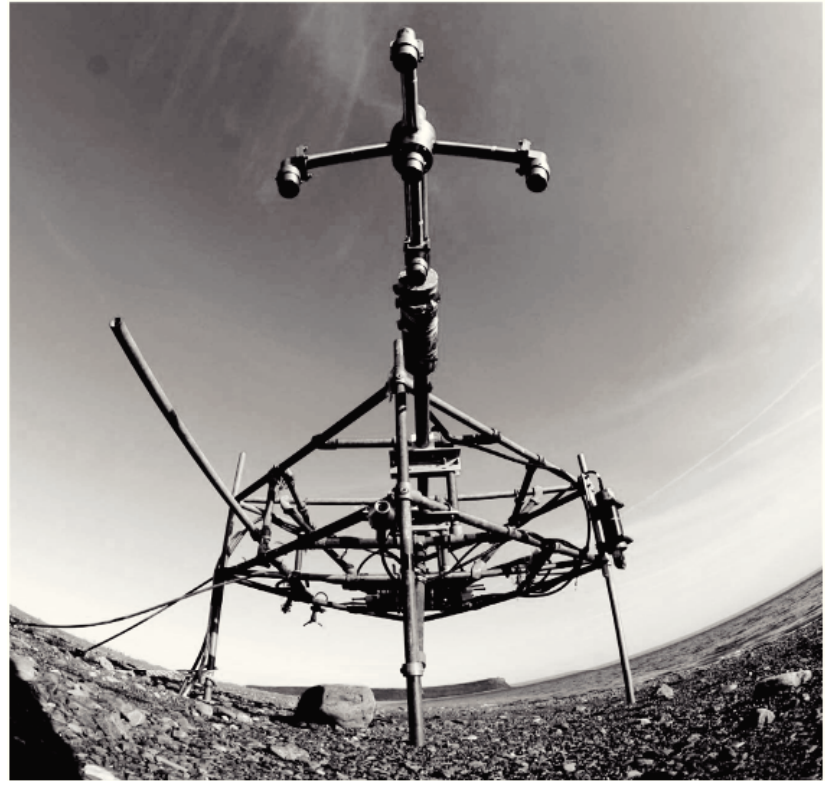

Figure 2. Fisheye view of the instrument frame, with the MFDop transducer assembly foremost. Note the wide range of bed material sizes.

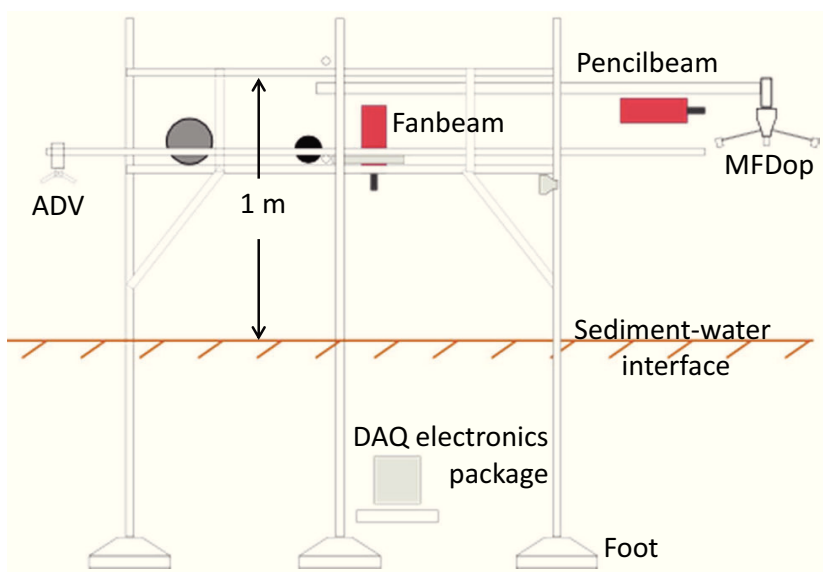

Figure 3. Instrument frame sketch, to scale: side view, facing seaward.

fixed to a plywood base. Size distributions were determined from the photographs using the autocorrelation method developed by Rubin (2004). The relatively small volume of the discrete samples, and the small $(20 \times 40 \mathrm{~cm})$ area of the photographs were such that reliable estimates of the cobble size fraction were not obtained. The overall average size distribution - based on all samples from the active part of the beach face - was unimodal but highly skewed toward the larger sizes, as reflected by the values of $D_{16}, D_{50}$, and $D_{84}$ (percent coarser), which were $17.2,4.2$, and $1.0 \mathrm{~mm}$ respectively. Further details on the sediment sampling and size analysis methodologies used in the 2012 experiment are given in Stark et al. (2014).

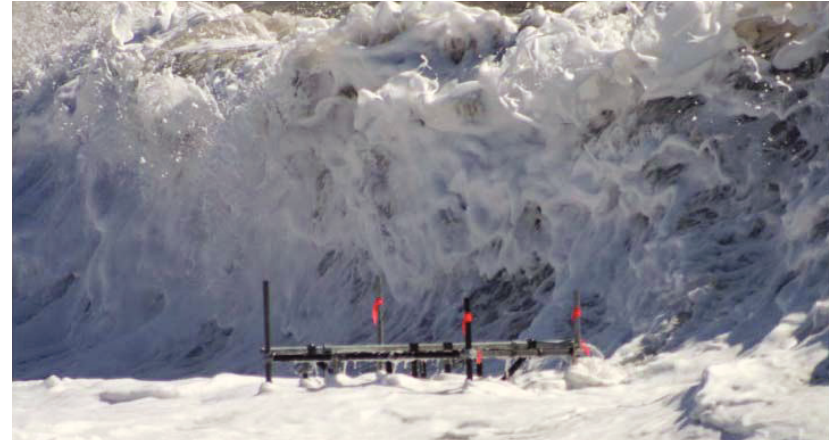

Figure 4. The instrument frame in an energetic shore break.

The 10-12 m tidal range, and the midtide location of the instrument platform, meant that the support structure for the platform would be subjected to non-negligible forces during significant wave forcing events. These forces were especially severe when the shore break passed the platform location on the rising or falling tide, as illustrated by the photograph in Fig. 4. It was also important that the frame legs be as thin as possible so as to minimize their effect on the local sediment dynamics. Consequently, the frame was constructed from $3.4 \mathrm{~cm}$ diameter steel pipe, and each of the four legs was bolted to a $100 \mathrm{~kg}$ foot buried about $1 \mathrm{~m}$ below the sedimentwater interface (Fig. 3). The pressure housing for the data acquisition (DAQ) system was also buried, to minimize the total surface area of the exposed part of the platform. Power and communications - Ethernet and serial - to the DAQ were provided via buried cables.

\section{Results}

\subsection{Forcing conditions}

Time series of the primary forcing parameters as registered by the ADV are plotted in Fig. 5: (a) water depth, $h$; (b) significant wave height, $H_{1 / 3}=4 \sigma_{p}$, where $\sigma_{p}$ is the rms (root mean square) pressure, in metres, in the wind waveband (Thornton and Guza, 1983); (c) significant wave orbital velocity amplitude is similarly defined as $V_{1 / 3}=2 \sigma_{V}$, where $\sigma_{V}$ is the square root of the sum of the variances of two horizontal velocity components in the waveband; and (d) the wave angle of incidence, $\alpha$. Typical peak periods were short - between 5 and $7 \mathrm{~s}-$ so the wind waveband was defined to be $0.05-0.7 \mathrm{~Hz}$, the latter higher than the usual $0.3 \mathrm{~Hz}$ because of the high frequency content of the (fetch-limited) wave spectrum at Advocate Beach. Mean currents (not shown) were weak: less than $0.25 \mathrm{~m} \mathrm{~s}^{-1}$ alongshore, and $0.07 \mathrm{~m} \mathrm{~s}^{-1}$ cross-shore. Noteworthy in relation to the results for bed roughness response presented later, the alongshore current maxima coincided with high tide, and alongshore currents were less than $0.08 \mathrm{~m} \mathrm{~s}^{-1}$ immediately after the shore break passed the instrument location on the rising tide. 

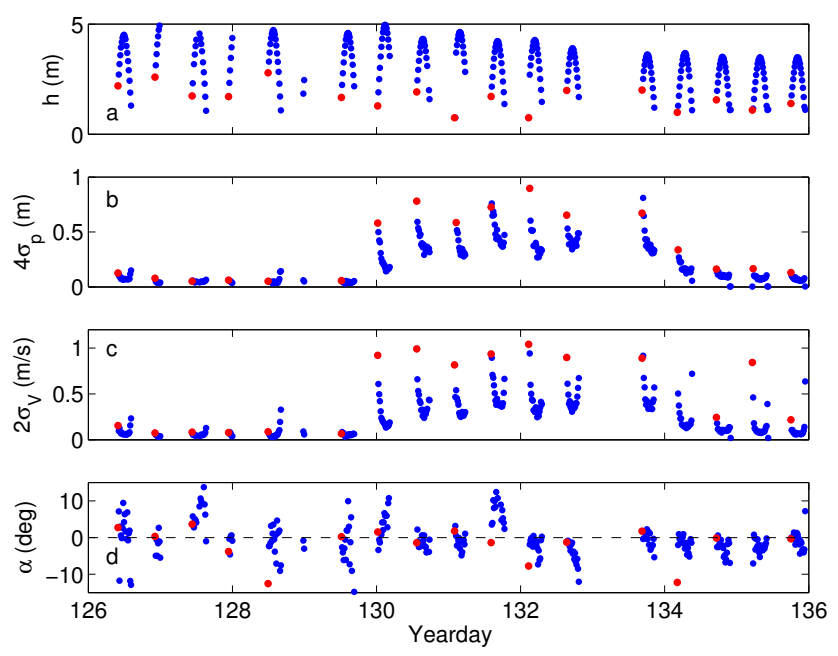

Figure 5. ADV time series: (a) mean water level; (b) significant wave height, $4 \sigma_{p}$; (c) significant wave orbital velocity, $2 \sigma_{v}$; (d) wave angle of incidence. The first data points on the rising tide are red.

Conditions were relatively calm until (year day) YD130, when a wave forcing event lasting 4 days occurred (Fig. 5b, c). Significant wave heights and wave orbital velocities reached $1 \mathrm{~m}$ and $1 \mathrm{~m} \mathrm{~s}^{-1}$ respectively, with the highest values typically occurring on the rising tide in each case. The wave angle of incidence was near zero at these times, i.e. near normal incidence.

A data gap is apparent in the time series in Fig. 5: there are no data for the first tide on YD133. A major difficulty in making measurements in the intertidal zone on a steep beach with coarse-grained sediments is the risk of damage to the instrumentation from impacts with quite massive particles i.e. gravel and possibly even cobbles - moving at velocities of metres per second. At Advocate Beach this difficulty is compounded by the prevalence of floating debris, including lumber from undermined shoreline structures, but the most serious risk was from logs of up to $5 \mathrm{~m}$ or more in length, and up to $30 \mathrm{~cm}$ or more in diameter. The top of the dune, which is occasionally overtopped during severe winter storms, is littered with this material. During the experiment, a daily activity was to walk the length of the beach at low tide to remove logs and wood debris. However, at the height of the storm on YD132 with the tide rising, several large logs floating nearby and nightfall descending, we elected to remove the MFDop from the frame. In making the necessary changes to the data acquisition protocol the next tide (i.e. the first on YD133) was missed.

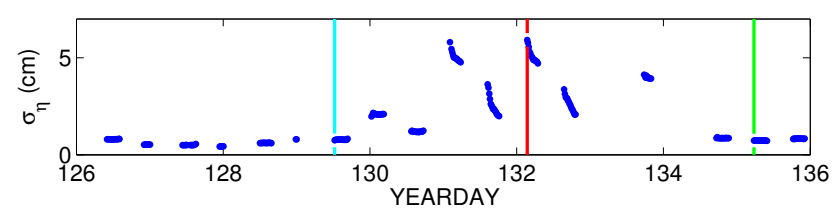

Figure 6. Rms bed roughness, $\sigma_{\eta}$, from the bed profiles measured with the rotary pencil-beam sonar. Compare to the forcing time series (Fig. 5). The vertical cyan, red and green lines correspond to the times indicated in Fig. 7.

\subsection{Bed roughness response}

The time series of rms bed roughness, $\sigma_{\eta}$ - determined via the bed profiles extracted from the rotary pencil-beam sonar imagery (see Hay, 2011, and references therein for data processing details) - at the instrument frame location is presented in Fig. 6. Prior to the onset on YD130 of the 4-day wave event, $\sigma_{\eta}$ was small, with values ranging from 0.5 to $1 \mathrm{~cm}$, the variations being largely due to the presence/absence of cobbles - see Fig. 2 - along the $4 \mathrm{~m}$ long cross-shore sweep of the sonar beam. As Fig. 6 demonstrates, a pronounced increase in the values of $\sigma_{\eta}$-by as much as an order of magnitude - occurred during the wave event. Notably, the highest rms roughness values occurred on the rising tide. After the wave forcing died out at midday on YD134 (Fig. 5b, c), $\sigma_{\eta}$ returned to pre-event levels.

The increased rms roughness during the wave forcing event was due to the formation of up to $1.5 \mathrm{~m}$ wavelength, up to $20 \mathrm{~cm}$ height ripples, as demonstrated by the pencilbeam bed profiles in Fig. 7 and the fan-beam image in Fig. 8. There is a pronounced difference between ripple crest and trough in the texture of the fan-beam image, indicating that surficial sediments in the crests were composed of much finer sediment than that in the troughs, which included gravel and cobble-sized material of up to $20 \mathrm{~cm}$ in diameter. The inference is that the coarse material in the troughs is a lag deposit, created by the gathering - via wave action - of the finergrained and thus more mobile sand-sized material into the ripple crests (see Sect. 4).

As indicated by the roughness time series in Fig. 6, ripple formation occurred on the rising tide. The actual formation process was not captured in this data set, either because the rotary sonar transducers were not yet immersed in water - being about $0.5-1 \mathrm{~m}$ above the bed surface (Fig. 3) - or because bubbles from breaking waves obscured the seabed. The rapid rise rate of mean water level $\left(3 \mathrm{~m} \mathrm{~h}^{-1}\right.$ at midtide) places a stringent constraint on the ripple formation time (see Sect. 4).

The roughness time series also indicates that after initial formation, the ripples gradually decayed under the action of the waves as the mean water level continued to rise to high water and then fall. Importantly, the ripples were still present during the ebb up until the point that the sonars could no longer detect the bed. Figure 9 illustrates the persistence 


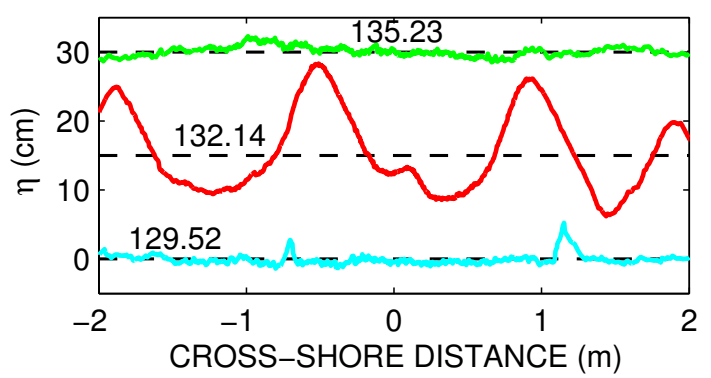

Figure 7. Selected profiles of bed elevation, $\eta$, with the bed slope removed. The numbers (in black) above each profile indicate the corresponding year day (see the roughness time series in Fig. 6). Successive profiles are offset by $15 \mathrm{~cm}$ in the vertical. Cross-shore distance is positive offshore.

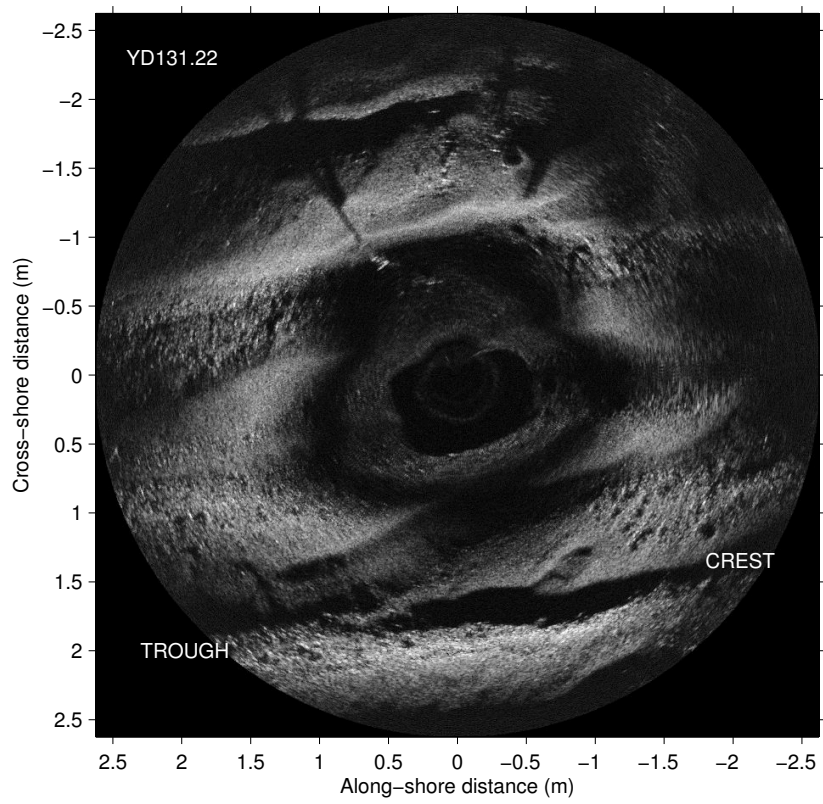

Figure 8. Rotary fan-beam sonar image, showing metre-scale wavelength ripples formed on the rising tide. Note the pronounced textural difference between ripple troughs and crests, and the cobbles of up to $10-20 \mathrm{~cm}$ diameter in the ripple troughs (e.g. at lower right). Lighter shades of gray correspond to higher amplitude backscatter. Cross-shore distance is positive offshore.

of the ripples, with little change in wave length despite the decay in ripple amplitude. Note too the slight offshore migration - by about a $1 / 4$ wavelength - during the hour after the first profile. This short duration, short distance migration - also observed in the fan-beam images - was typical: migration distances longer than half a wavelength were not observed.

\subsection{Beach-face grain-size response}

Significant changes in the grain-size distribution of the surficial sediments were observed during the course of the ex-

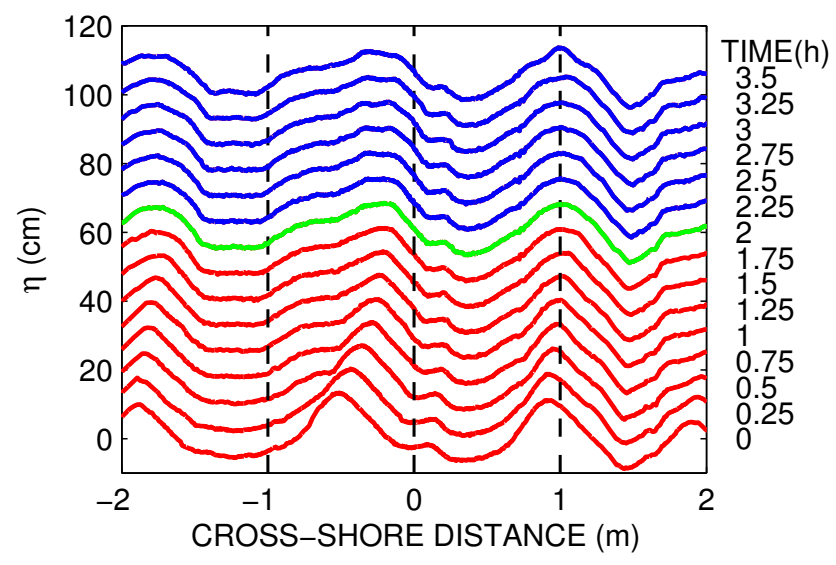

Figure 9. Ripple profile time stack during the first tide on YD132. Red indicates rising tide, green high tide, blue ebb. Cross-shore distance is positive offshore.

periment. As indicated by the time series of photographic estimates of mean size (Fig. 10), a pronounced minimum in the median grain size of the surficial sediments - averaged over the lower two-thirds of the beach face - coincided with the wave event. Grain size based on the sieve analysis indicates a similar fining of the surficial sediments during more energetic wave forcing. The gravel and sand size fractions by weight, based on samples collected along the same crossshore distance interval as the photographic results in Fig. 10 and the Udden-Wentworth scale, were 74 and $26 \%$ respectively on YD125 (10 samples), and 48 and $52 \%$ respectively on YD131 (8 samples).

Thus, perhaps counter-intuitively, energetic wave forcing led to fining of the beach-face material. This result is surprising, as finer material is typically removed from a beach during the initial stages of a storm and during the winter storm season (e.g. Komar, 1998, Sect. 7). Given the bed roughness results in the previous section, the clear inference is that the fining of the surficial sediments was associated with the occurrence of the ripples. In Sect. 4 the fining is attributed to infilling of the troughs with the fine material in the crests as part of the planing-off process by the shore break and swash during the falling tide.

\subsection{MFDop results}

\subsubsection{Vertical structure}

One of the main goals of the Advocate Beach experiment was the deployment of the MFDop in the field for the first time. A primary scientific objective was to measure the vertical structure of the wave orbital motion, including the WBBL, because of the pronounced difference in the theoretically predicted vertical structure of the wave shear stress above a sloping bed in breaking and non-breaking wave conditions (Rivero and Arcilla, 1995; Zou et al., 2006). 


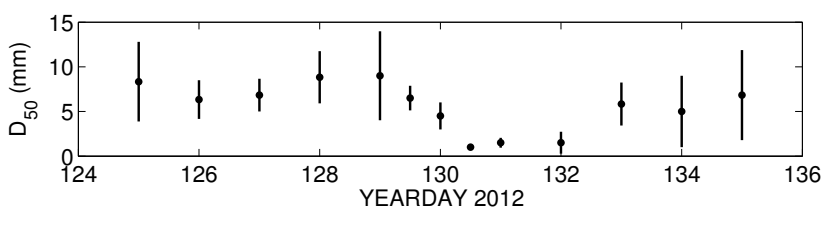

Figure 10. Grain size versus time. The points are the values of $D_{50}$ averaged at 6 locations evenly spaced across the shore face, from $15 \mathrm{~m}$ shoreward to $45 \mathrm{~m}$ seaward of the frame location. Compare to the forcing and bed roughness time series (Figs. 5, 6). The plotted grain sizes were obtained using the Rubin (2004) method.

The vertical structure of the oscillatory motion in the waveband is presented in Fig. 11. The expected phase relationship between the cross-shore velocity, $v(-v$ is plotted so as to be positive shoreward), and the vertical velocity $w$ (positive up) is evident; i.e. close to the bed, $v$ and $w$ are in phase, necessitated by the bottom slope and the condition of no flow normal to the bed so $w=v \tan \beta$ ( $\beta$ being the bed slope). Farther from the bed $v$ and $w$ tend toward quadrature (Fig. 12a) with shoreward velocity leading $w$, as expected for shoreward propagating waves. The magnitude of the corresponding wave shear stress is shown in Fig. 12b: the nearbed peak due to vertical turbulent momentum flux within the WBBL, and the decay of $\langle v w\rangle$ as height above the bed increases, are both consistent with theoretical predictions (Zou et al., 2006).

The data in Fig. 11 correspond to a time of weak wave forcing when the bed was nominally flat (Figs. 6, 7). As the existing theory has been developed for a flat bed only, however, the development of the large-amplitude ripples during energetic conditions has so far precluded comparisons between theory and experiment under breaking waves within the context of this data set.

\subsubsection{Wave bottom boundary layer turbulence}

The MFDop is designed to be turbulence-resolving in order to obtain estimates of the Reynolds stress in the WBBL. The vertical velocity spectral density, $S_{\mathrm{ww}}$, at $3 \mathrm{~cm}$ height above bottom is plotted in Fig. 13. Three spectra are shown. Because of the five-transducer geometry of the MFDop (Fig. 2), three independent measurements of the vertical velocity are obtained: one from the centre transducer, and one from each of the two opposed outboard transducer pairs. The spectra in Fig. 13 exhibit a well-defined inertial subrange. The noise level in the spectrum for the vertical beam is noticeably higher: this is because the signal level from the centre transducer is attenuated by the transmit/receive switch in the analog electronics.

\subsubsection{Bed material velocity}

The MFDop was also developed to measure the velocity of material moving on the bed surface - i.e. the velocity of bed-
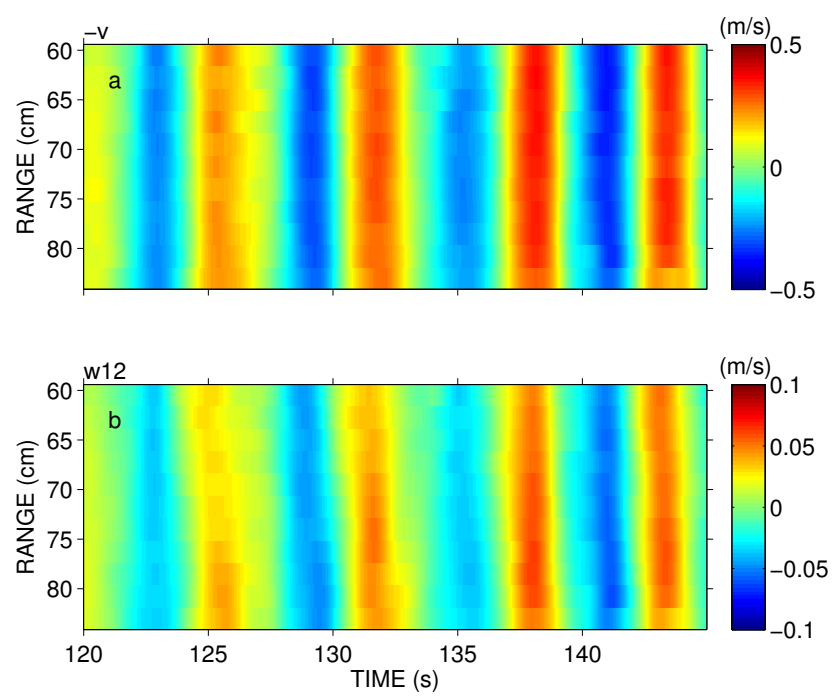

Figure 11. Waveband velocity profiles from the MFDop: (a) crossshore velocity, positive onshore; (b) vertical velocity, positive up. The bottom is the zero-velocity band starting at about $83 \mathrm{~cm}$ range. Note the transition in the $v$ vs. $w$ phase relationship with range, i.e. from quadrature far from the bed to in phase close to the bed. Data are from YD130.64, i.e. when the bed was quasi-flat prior to ripple formation (see Fig. 6). See Fig. 13 for the meaning of w12.
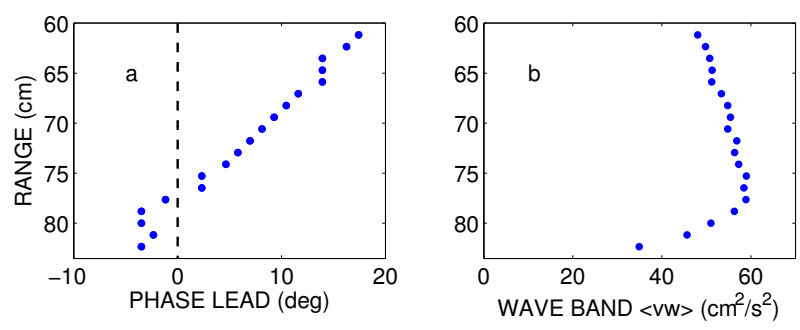

Figure 12. Vertical structure of the phase and magnitude of the waveband cross-shore shear stress. YD130.64.

load material - and ultimately the bedload transport. The poorly sorted nature of the bed material at Advocate Beach is such that estimating the transport was not tractable. The velocity of the bed material was measured, however, as Fig. 14 demonstrates. Plotted in Fig. 14 are (a) net along- and crossshore bed material displacements - i.e. the time integral of the bed velocities from the MFDop - over the $260 \mathrm{~s}$ length of the record; (b) the time series of $u$ and $v$ outside the WBBL as registered by the Vector ADV; and(c) the cube of the envelope - i.e. the amplitude $v_{0}$ - of the cross-shore velocity, obtained via the Hilbert transform of $v$ in panel $b$. There is a clear relationship between $v_{0}^{3}$ and the times of significant cross-shore displacement of bed material. 


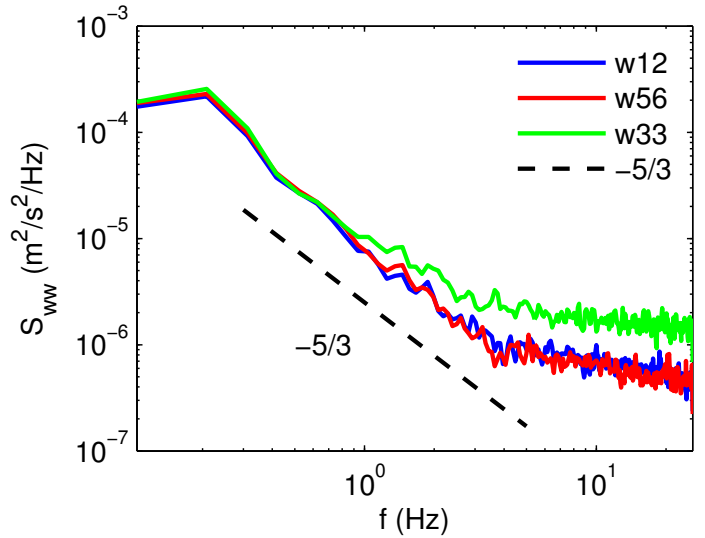

Figure 13. Vertical velocity spectra at $80 \mathrm{~cm}$ range - i.e. $3 \mathrm{~cm}$ above the bed - illustrating the well-resolved inertial subrange in the WBBL. Green indicates $w$ from the centre transducer, red and blue $w$ from the two orthogonal outboard pairs. YD130.64.
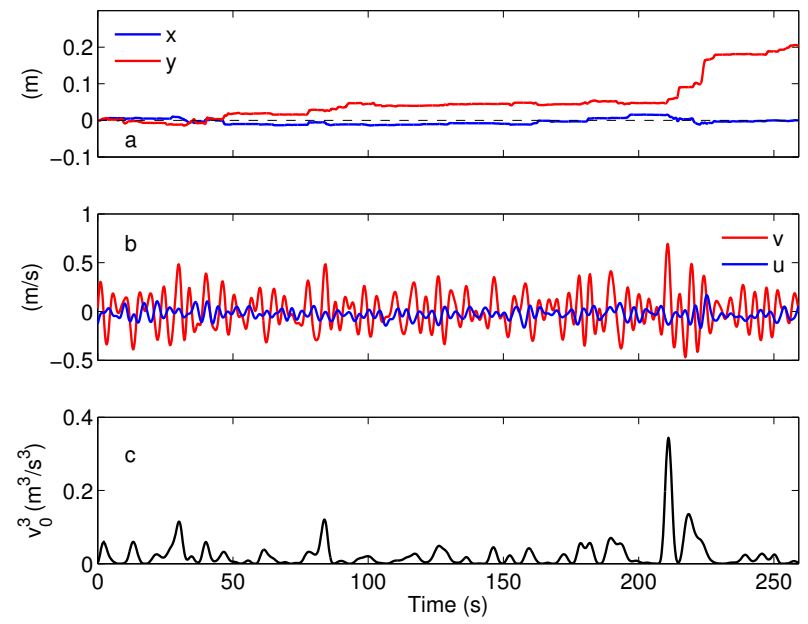

Figure 14. (a) Cross-shore $y$ and along-shore $x$ bed material displacements, from the time integral of the MFDop velocities at the range corresponding to peak backscatter from the seabed. (b) Crossshore $v$ and along-shore $u$ velocities outside the WBBL, from the ADV. (c) The cubed envelope of $v$ (see text).

\section{Discussion}

We now return to the connection(s) between grain-size segregation during ripple formation and the decrease in the median diameter of the beach-face sediments during energetic wave forcing. There are eight main points to consider: (1) the ripples formed rapidly; (2) they are orbital-scale ripples; (3) the energetic shore break; (4) the very broad sediment size distribution; (5) the always planar beach face at low tide; (6) the $\mathrm{O}(10 \mathrm{~cm})$ thickness of the fine sediment veneer; (7) the constant mean elevation of the beach face at the frame location; and (8) the constant height of the pencil beam above mean bed level.
The rate of water level rise at midtide was $3 \mathrm{~m} \mathrm{~h}^{-1}$, so the length of time between mean water level arriving at the frame location and then rising to the $1 \mathrm{~m}$ height of the pencil-beam sonar would have been $20 \mathrm{~min}$. Thus, no more than 20 to perhaps $30 \mathrm{~min}$ were available for the ripples to form.

Orbital-scale ripples - sometimes called vortex ripples are characterized by wavelengths, $\lambda$, linearly proportional to the near-bed wave orbital excursion, $d_{0}$; i.e. $\lambda=K d_{0}$. With $U_{0}$ being the near-bed orbital velocity amplitude and $T_{p}$ the peak wave period, $d_{0}=U_{0} T_{p} / \pi$. For newly formed equilibrium orbital ripples $K$ is typically between 0.6 and 0.7 (Nielsen, 1981; Clifton and Dingler, 1984; Traykovski et al., 1999 , many others). The steepness $\eta_{0} / \lambda$ - with $\eta_{0}$ being the ripple height - is ca. 0.2. The characteristics of the ripples in the present study are listed in Table 1 . The values of $\lambda$ and $\eta_{0}$ are from the first bed profile on the rising tide, and are based on a minimum of four contiguous ripples in each profile. Data are listed for only those rising tides for which the within-profile variation in ripple wavelength was less than $20 \%$, as indicated by the standard deviations in Table 1 . Also listed in Table 1 are the forcing conditions - i.e. the wave peak period (from the peak in the cross-shore velocity power spectrum) and orbital velocity amplitude - corresponding to the first ADV measurement on the rising tide, i.e. the red points in Fig. 5.

The observed steepnesses are ca. 0.13 , so somewhat low but within the expected range for irregular waves (Nielsen, 1981). The observed wavelengths were comparable to - but longer than - the expected equilibrium value, the values of $K$ averaging to 0.81 . However, the ADV was located $68 \mathrm{~cm}$ above the beach face (Fig. 3), and may therefore have been out of the water or suffered from bubble contamination during the initial stages of ripple formation. Thus the values of $U_{0}$ appropriate for ripple scaling may be higher than those listed in Table 1. In principle, linear wave theory could be invoked to correct $U_{0}$ using some mean water depth at the time of ripple formation, but neither is this time known, nor is it clear that such a mean water depth would be meaningful. The $20 \mathrm{~min}$ formation time and $5 \mathrm{~s}$ typical wave period correspond to only 240 wave cycles, and during this time the wave orbital velocity at bed level would not have been quasi-steady due to the rapidly changing water depth. Consequently, the ripples were unlikely to have been in equilibrium with the forcing during formation, possibly accounting for the somewhat low steepness and long wavelength, though the gravel lag in the troughs and other mixed grain-size effects are likely important (Note that recent measurements in an oscillatory water tunnel by Cummings et al. (2009) have shown - for equilibrium vortex ripples - that while $K \sim 0.6$ in fine sand, $K \sim 0.4$ in coarse sand. Thus, if the representative value of $U_{0}$ during ripple formation at Advocate Beach were to be significantly greater than $1 \mathrm{~m} \mathrm{~s}^{-1}$ as suggested above, the corresponding increase in $d_{0}$ would bring our estimates of $K$ closer to the Cummings et al. (2009) value.). 
Table 1. Ripple properties and associated wave forcing parameters.

\begin{tabular}{cccccccccc}
\hline YD & $\begin{array}{c}\lambda \\
(\mathrm{m})\end{array}$ & $\begin{array}{c}\eta_{0} \\
(\mathrm{~cm})\end{array}$ & Crests & Troughs & $\eta_{0} / \lambda$ & $\begin{array}{c}U_{0} \\
\left(\mathrm{~m} \mathrm{~s}^{-1}\right)\end{array}$ & $\begin{array}{c}T_{p} \\
(\mathrm{~s})\end{array}$ & $\begin{array}{c}d_{0} \\
(\mathrm{~m})\end{array}$ & $\lambda / d_{0}$ \\
\hline 131 & $1.30 \pm 0.13$ & $18 \pm 0.7$ & 3 & 4 & 0.14 & 0.82 & 5.7 & 1.47 & 0.89 \\
132 & $1.27 \pm 0.22$ & $17 \pm 2.7$ & 4 & 4 & 0.13 & 1.04 & 4.2 & 1.41 & 0.91 \\
133 & $0.90 \pm 0.16$ & $12 \pm 2.0$ & 5 & 5 & 0.13 & 0.89 & 4.9 & 1.37 & 0.65 \\
\hline
\end{tabular}
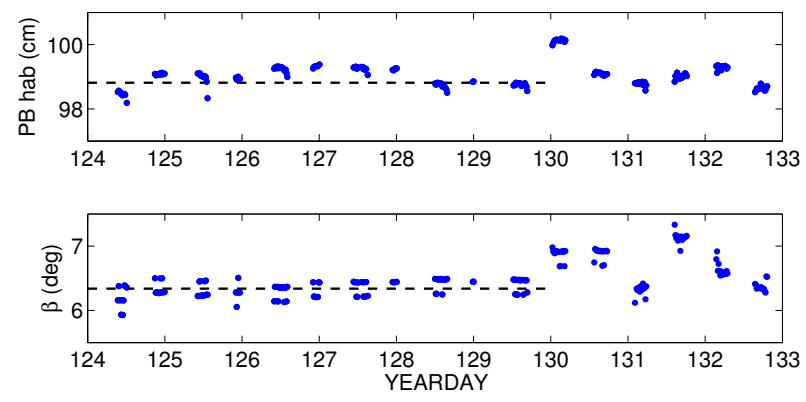

Figure 15. (a) Pencil-beam height above mean bed level. The dashed line indicates the $98.8 \mathrm{~cm}$ pre-storm average height. (b) Local bed slope, $\beta$, from the pencil-beam profiles. The quantization of the $\beta$ values within a given tidal cycle is due to the $0.225^{\circ}$ rotary sonar step size. The dashed line indicates the $6.3^{\circ}$ pre-storm average.
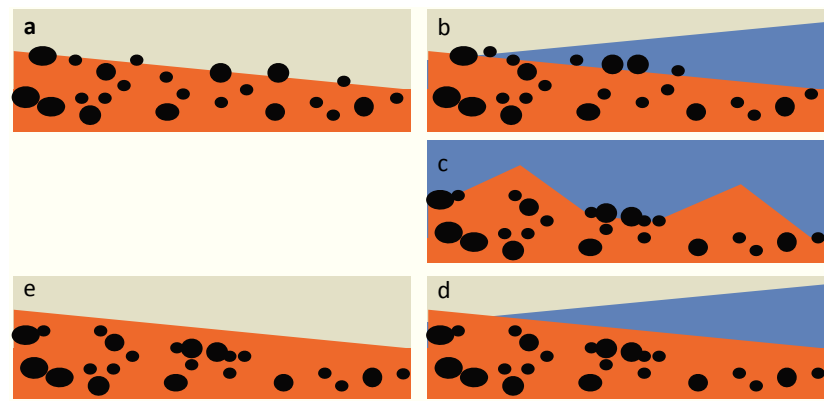

Figure 16. Cartoon illustrating the sequence leading to the surficial fine-grained sediment layer via the development of vortex ripples. Cobbles and stones are black, finer sediments brown. (a) Initial state at low water. (b) Rising tide, larger particles displaced/dislodged by the shore break and swash. (c) Rising tide after passage of the shore break and swash, finer sediments have been gathered into ripple crests, leaving a stone and cobble lag in the troughs. (d) Falling tide, ripples planed flat by swash and shore break, creating a veneer of fine sediment. (e) Final state at low water.

The height of the pencil-beam sonar above mean bed level is plotted in Fig. 15a. Each point represents the vertical distance from the sonar head to the least-squares best-fit straight line through the bed profile within $\pm 2.3 \mathrm{~m}$ of the sonar's cross-shore location. (This best-fit line was subtracted from the measured profile to obtain the bed elevation profiles with the slope removed in Fig. 7, and the rms bed roughness values in Fig. 6.) The values of bottom slope, $\beta$, obtained from the best fit are shown in Fig. 15b, indicating that the local bed slope remained essentially constant at its $6.3^{\circ}$ average value prior to the storm, and changed by at most $0.5^{\circ}$ during the storm. The data points in Fig. 15a demonstrate that the height of the pencil beam above mean bed level remained essentially constant, varying by less than $1 \mathrm{~cm}$ even during the rippled bed interval, YD130-133. Simulations (not shown) - with $1-1.5 \mathrm{~m}$ wavelength, $20 \mathrm{~cm}$ high sinusoidal ripples on a $4.6 \mathrm{~m}$ long $6.5^{\circ}$ slope centred at $x=0$, and $z=-1 \mathrm{~m}$ - indicate that the $1 \mathrm{~cm}$ variations in height and $0.5^{\circ}$ variations in bed slope during the storm interval can be attributed to the non-integral number of ripples within the $4.6 \mathrm{~m}$ long profile. The implication is that - to a first approximation sediment was locally conserved; i.e. the ripples were built up mainly from local sediments and then, when later planed flat, the troughs were infilled mainly with the sediments from the nearby crests.

The fact that as little as $20 \mathrm{~min}$ are available for ripple formation indicates that the fine-grained material in the ripple crests could not have come from elsewhere. The source had to be the local beach face. Thus, as illustrated in Fig. 16 and described below, a four-stage scenario is indicated involving: (I) non-size-selective sediment mobilization by the shore break, (II) ripple formation, (III) partial decay, and (IV) erasure. (I) Mobilization: it is highly likely that the intense shore break played an important role, generating forces sufficient to produce movement of the larger stones as well as the finer material. We do have evidence - from a second experiment in October-November 2013 - of the swash and shore break displacing stones and cobbles. It is also possible that a step in the beach profile - a common feature at the shore break on steep beaches (e.g. Buscombe and Masselink, 2006) - was a contributing factor in the development of the ripples (see last paragraph, this Section), although we have no direct evidence of a step being present. Regardless of the presence/absence of a step, the physical picture is of the shore break advancing rapidly up the beach face with the rising tide, and mobilizing sediment of all sizes. (II) Formation: after the passage of the shore break, the ripples would have started to form. Typically, vortex ripples develop from smaller ripples (Bagnold, 1946; Sleath, 1984, many others). Once the lee vortex forms, growth continues as the vortex sweeps material out of the troughs toward the crests. In our case, material too big to be mobilized by the turbulent stresses in the lee vortex would have remained in the troughs, creating the 
cobble lag seen in the sonar images. At this point growth would have stopped, providing an additional reason for the somewhat low steepness, i.e. crests starved of sediment supply. (III) Decay: as the flood progressed the ripple amplitudes gradually decayed, by as much as $50 \%$ (Fig. 6). This decay was due to depth-controlled reduction in wave forcing at the bed - the associated near-bed orbital excursion would have been incompatible with the scale of the ripples - and the background turbulence associated with the waves and mean current (which peaked at high tide). The ripples did not rebuild on the ebb. (IV) Erasure: as the swash and shore break passed the frame again on the ebb, the ripple crests were planed off, and the troughs infilled with fine sediment, resulting in the layer of fine sediment on the beach face. The observed thickness of this layer was $5-10 \mathrm{~cm}$, consistent with the $20 \mathrm{~cm}$ trough-to-crest height of the ripples.

Laboratory studies of vortex ripple formation in heterogeneous sediments have been carried out by Foti and Blondeaux (1995, FB95) and by Rousseaux et al. (2004, R04). Binary mixtures of sand-size (glass) particles were used: 0.65 and $1.5 \mathrm{~mm}$ diameter (FB05); and $0.15-0.355 \mathrm{~mm}$ (R04). The finding in both studies was that coarser-grained material accumulated at the ripple crests, not in the troughs. However, the larger and smaller particle diameters differed by less than a factor of 3 , compared to the factor of 200 between the millimetre-sized sand and $20 \mathrm{~cm}$ diameter cobbles at Advocate Beach. It is clear that the coarsest sediment in the FB05 and R04 experiments was mobile during ripple formation, whereas our observations indicate that the stone/cobblesized material was likely not (i.e. certainly not once the shore break had passed the frame location at least).

Segregation of fine/coarse material in the crests/troughs of dunes in unidirectional flow - i.e. "downward coarsening" dates back to Bagnold in 1941, and was recently investigated in a flume experiment by Blom et al. (2003). Three points regarding the latter study are particularly relevant here: (1) the grain-size distribution was broad, a factor of 10 separating the large and small particle diameters, and so more comparable to Advocate Beach than the FB05 and R04 studies; (2) coarse-grained layers formed at trough level beneath the migrating dunes; and (3) sweeping of finer material from the troughs by turbulence in the lee vortex was suggested as a contributor to the coarse lag in the dune troughs.

We briefly consider the possible role of hydraulic conductivity, which Mason and Coates (2001) considered "perhaps the most distinctive property which distinguishes a mixed beach". In the Advocate Beach 2012 experiment a pressure sensor was buried in the beach at the instrument frame location. The data (not shown) indicate a pronounced flood/ebb asymmetry such that, on the ebb, elevated pore pressures persisted for $\mathrm{O}(1 \mathrm{~h})$ after the mean water level had receded past the frame. This asymmetry is due to slow exfiltration of the groundwater which had penetrated the beach face on the preceding flood. We speculate that a contributing factor to ripple erasure during the ebb might have been reduced internal fric- tion within the sediments due to the upward flow of groundwater out of the beach face.

A mechanism is required to account for the disappearance of the fine sediment veneer as the wave forcing waned at the end of the storm. The data in Fig. 10 indicate that this process occurred within a tidal cycle. One possibility might be the Brazil nut effect, in which smaller particles fall down into pores created when the sediment is shaken bodily, forcing larger grains to rise to the top. However, it is not obvious that this effect would operate when the forcing is a shear stress applied at the top of the sediment column. We suggest instead, as a hypothesis, that infiltration on the rising tide may play a key role. One possibility is that finer-grained material is suspended in the swash and then drawn with the infiltrating water into the spaces between the now-exposed coarser grains. It is also possible that the finer grains are drawn directly from the surface of the beach -i.e. without being resuspended - into voids within the sediment matrix as the comparatively still water at the top of the uprush infiltrates the beach.

Finally, we return to the question of the presence/absence of a breakpoint step at Advocate Beach. In the Ivamy and Kench (2006) study of an MSG beach, a pronounced $1 \mathrm{~m}$ high breakpoint step persisted throughout the $9 \mathrm{~d}$ duration of the experiment. The step migrated shoreward on the flood, and back to its original position on the ebb. The migration distance was proportional to the tidal range - i.e. the $14 \mathrm{~m}$ maximum observed onshore migration corresponds to the $1.4 \mathrm{~m}$ maximum tidal range and the roughly $1: 10$ beach slope. IK06 measured depths of disturbance exceeding $0.5 \mathrm{~m}$ at the step, definitive evidence that the breakpoint on a steep beach is a region of highly active sediment transport, consistent with the mobilization phase here (i.e. Fig. 16b). Intriguingly, IK06 also mention bedforms seaward of the breakpoint on the rising tide: "Visual observations showed this process generated undulating bedforms of mixed sand and gravel to the limit of breakpoint step migration (Fig. 10a, b)". The "process" IK06 refer to is the migration across the beach face of intense sediment reworking in the breaker zone. The IK06 beach was exposed to Pacific Ocean swell, so waves were breaking on the beach even between their two wind-wave events, and hence the ever-present step. In contrast, Advocate Beach faces the fetch-limited Bay of Fundy/Gulf of Maine system and is therefore seldom subjected to ocean swell, and was not during the 2012 experiment (as indicated by the low waveband orbital velocities in Fig. 5). Thus a step was not present at low tide level during the time periods with weak wind-wave forcing. Although we have no direct evidence of a step being present during the storm, it is conceivable that one was - but possibly nascent given the rapidity of the shore break advance up the beach face - and passed the frame location before the sonars were able to detect the bed. If the IK06 undulating bedforms are taken to be orbital (vortex) ripples, their visual observations suggest that the ripples at Advocate Beach might have formed in the wake of a (possibly 
nascent) step associated with the shoreward-migrating breakpoint. Then the usual initial stages in vortex ripple development from a flat bed - the formation and subsequent coalescence through wavelength doubling of short-wavelength low-amplitude ripples - would have been short-circuited. Instead, ripples would form at a scale commensurate with the amplitude and steepness of the nascent step, a scale necessarily much larger than that of small-scale rolling grain or anorbital ripples (Bagnold, 1946; Sleath, 1984). Thus, with the migrating (nascent) step being the initial perturbation of an otherwise flat bed, vortex ripples would have formed much more rapidly than otherwise.

\section{Summary and conclusions}

During active transport conditions dominated by wind-wave forcing, ripples of up to $1.5 \mathrm{~m}(20 \mathrm{~cm})$ wavelength (height) were observed to form at the midtide level. Textural differences in the rotary sonar imagery indicate that the ripple crests were composed of fine-grained sediment, while the ripple troughs were lined with gravel and cobbles of up to $20 \mathrm{~cm}$ in diameter. We infer that the finer material was gathered into the ripple crests, leaving a gravel-and-cobble lag in the troughs. Following their formation on the rising tide, the ripples persisted - though with decreasing amplitude - until the last observations on the falling tide when the instruments were no longer continuously submerged. At low tide, when the beach was again fully exposed, the ripples were no longer present, and the beach face had returned to its planar state. We conclude that the ripple crests had been planed flat by the high bed shear stresses under the swash and shore break.

The grain size of the surficial sediments in the intertidal zone, sampled at low tide when the beach face was fully exposed, exhibited a pronounced minimum coinciding in time with maximum wave-forcing: the average median diameter was reduced from that during calm conditions by a factor of 4. We conclude that the flattening of the ripple crests by the swash/shore break during ebb was accompanied by infilling of the troughs with the finer-grained crest material, resulting in a uniform, $\mathrm{O}(10 \mathrm{~cm})$ thick veneer of sand-sized sediment and return to a planar beach face at low tide.

The MFDop results are promising. The vertical structure of waveband motions exhibits the features predicted by theory over a sloping bed: the transition from vertical and crossshore velocities being in quadrature far from the bed to inphase as the bed is approached; a near-bed maximum in the wave shear stress associated with the wave bottom boundary layer; and - under non-breaking waves - a linear decay in the wave shear stress with height above the bed. Vertical velocity spectra exhibit a well-defined inertial subrange indicative of turbulence within the wave-bottom boundary. Finally, the measurements of the velocity of material at bed level indicate a $u^{3}$ dependence and, thus, promise for field measurements of bed material transport in the future.
Acknowledgements. This work was funded by the Natural Sciences and Engineering Research Council of Canada, the Atlantic Innovation Fund, and Nortek. Richard Cheel, Walter Judge, Robert Craig and Doug Schillinger provided technical support and, together with D. Barclay, J. Hare, M. Hatcher, T. Guest and O. Lundesgaard, assistance in the field. The authors also wish to thank Evan Goldstein and an anonymous reviewer for their valuable comments, and the Editor Giovanni Coco for allowing us to extend the paper beyond its initial scope to include additional relevant material.

Edited by: G. Coco

\section{References}

Allan, J. C., Hart, R., and Tranquilli, J. V.: The use of Passive Integrated Transponder (PIT) tags to trace cobble transport in a mixed sand-and-gravel beach on the high-energy Oregon coast, USA, Mar. Geol., 232, 63-86, doi:10.1016/j.margeo.2006.07.005, 2006.

Bagnold, R. A.: Motion of waves in shallow water. Interaction between waves and sand bottoms, Proc. Roy. Soc. Lond., Ser. A 187, 1-15, 1946.

Bertoni, D., Sarti, G., Benelli, G., Pozzebon, A., and Raguseo, G.: Transport trajectories of "smart" pebbles on an artificial coarse-grained beach at Marina di Pisa (Italy): implications for beach morphodynamics, Mar. Geol., 291, 227-235, doi:10.1016/j.margeo.2011.08.004, 2012.

Bertoni, D., Grottoli, E., Ciavola, P., Sarti, G., Benelli, G., and Pozzebon, A.: On the displacement of marked pebbles on two coarse-clastic beaches during short fair-weather periods (Marina di Pisa and Portonovo, Italy), Geo-Mar. Lett., 33, 463-476, doi:10.1007/s00367-013-0341-3, 2013.

Blom, A., Ribberink, J. S., and de Vriend, H. J.: Vertical sorting in bed forms: Flume experiments with a natural and a trimodal sediment mixture, Water Resour. Res., 39, 1025, doi:10.1029/2001WR001088, 2003.

Buscombe, D. and Masselink, G.: Concepts in gravel beach dynamics, Earth-Sci. Rev., 79, 33-52, 2006.

Ciavola, P. and Castiglione, E.: Sediment dynamics of mixed sand and gravel beaches at short time scales, J. Coastal Res., SI 56, 1751-1755, 2009.

Clifton, H. E. and Dingler, J. R.: Wave-formed structures and paleoenvironmental reconstruction, Mar. Geol., 60, 165-198, 1984.

Cummings, D., Dumas, S., and Dalrymple, R. W.: Fine-grained versus coarse-grained wave ripples generated experimentally under large-scale oscillatory flow, J. Sed. Res., 79, 83-93, 2009.

Curtiss, G. M., Osborne, P. D., and Horner-Devine, A. R.: Seasonal patterns of coarse sediment transport on a mixed sand and gravel beach due to vessel wakes, wind waves, and tidal currents, Mar. Geol., 259, 73-85, 2009.

Dickson, M. E., Kench, P. S., and Kantor, M. S.: Longshore transport of cobbles on a mixed sand and gravel beach, southern Hawke Bay, New Zealand, Mar. Geol., 287, 31-42, 2011.

Foti, E. and Blondeaux, P.: Sea ripple formation: the heterogeneous sediment case, Coast. Eng., 25, 237-253, 1995.

Hay, A. E.: Geometric bottom roughness and the bed state storm cycle, J. Geophys. Res., 116, C04017, doi:10.1029/2010JC006687, 2011. 
Hay, A. E., Zedel, L., Cheel, R., and Dillon, J.: On the vertical and temporal structure of flow and stress in the turbulent oscillatory boundary layer above evolving sand ripples: Observations using a prototype wide-band coherent Doppler profiler, Cont. Shelf Res., 46, 31-49, 2012.

Ivamy, M. C. and Kench, P. S.: Hydrodynamics and morphological adjustment of a mixed sand and gravel beach, Torere, Bay of Plenty, New Zealand, Mar. Geo., 228, 137-152, 2006.

Jennings, R. and Shulmeister, J.: A field based classification scheme for gravel beaches, Mar. Geol., 186, 211-228, 2002.

Komar, P. D.: Beach Processes and Sedimentation, Prentice-Hall, Old Tappan, N.J., 1998.

Levoy, F., Anthony, E., Monfort, O., and Larsonneur, C.: The morphodynamics of megatidal beaches in Normandy, France, Geo. Mar. Lett., 171, 39-59, 2000.

Mason, T. and Coates, T.: Sediment transport processes on mixed beaches: a review for shoreline management, J. Coast. Res., 17, 645-657, 2001.

Miller, I. M. and Warrick, J. A.: Measuring sediment transport and bed disturbance with tracers on a mixed beach, Mar. Geol., 299302, 1-17, doi:10.1016/j.margeo.2012.01.002, 2012.

Miller, I. M., Warrick, J. A., and Morgan, C.: Observations of coarse sediment movements on the mixed beach of the Elwha Delta, Washington, Mar. Geol., 282, 201-214, doi:10.1016/j.margeo.2011.02.012, 2011.
Nielsen, P.: Dynamics and geometry of wave-generated ripples, J. Geophys. Res., 86, 6467-6472, 1981.

Rivero, F. J. and Arcilla, A. S.: On the vertical distribution of $\langle\tilde{u} \tilde{w}\rangle$, J. Coast. Eng., 25, 137-152, 1995.

Rousseaux, G., Caps, H., and Wesfreid, J.-E.: Granular size segregation in underwater sand ripples, Eur. Phys. J. E, 13, 213-219, 2004.

Rubin, D. M.: A simple autocorrelation algorithm for determining grain size from digital images of sediment, J. Sed. Res., 74, 2004.

Sleath, J. F. A.: Sea Bed Mechanics, John Wiley, New York, 335 pp., 1984.

Stark, N., Hay, A. E., and Zedel, L.: Sedimentological and morphological variations at a macro-tidal sandy gravel beach, Mar. Geol., in preparation, 2014.

Thornton, E. B. and Guza, R. T.: Transformation of wave height distribution, J. Geophys. Res., 88, 5925-5938, 1983.

Traykovski, P., Hay, A. E., Irish, J. D., and Lynch, J. F.: Geometry, migration, and evolution of wave orbital ripples at LEO-15, J. Geophys. Res., 104, 1505-1524, 1999.

Zou, Q., Bowen, A. J., and Hay, A.: Vertical distribution of wave shear stress in variable water depth: Theory and field observations, J. Geophys. Res., 11, C09032, doi:10.1029/2005JC003300, 2006. 